\title{
Changes of monocyte human leukocyte antigen- DR expression as a reliable predictor of mortality in severe sepsis
}

Jian-Feng $\mathrm{Wu}^{1+}$, Jie Ma ${ }^{1 \dagger}$, Juan Chen ${ }^{1}$, Bin Ou-Yang ${ }^{1}$, Min-Ying Chen ${ }^{1}$, Li-Fen Li ${ }^{1}$, Yong-Jun Liu' ${ }^{1}$ Ai-Hua Lin ${ }^{2}$ and Xiang-Dong Guan ${ }^{1 *}$

\begin{abstract}
Introduction: Many studies have shown that monocyte human leukocyte antigen-DR (mHLA-DR) expression may be a good predictor for mortality in severe septic patients. On the contrary, other studies found mHLA-DR was not a useful prognostic marker in severe sepsis. Few studies have taken changes of mHLA-DR during treatment into consideration. The objective of this study was to estimate the prognostic value of changes of mHLA-DR to predict mortality in severe sepsis.

Methods: In this prospective observational study, mHLA-DR was measured by flow cytometry in peripheral blood from 79 adult patients with severe sepsis. mHLA-DR levels were determined on day 0, 3, 7 after admission to the surgical intensive care unit (SICU) with a diagnosis of severe sepsis. $\triangle \mathrm{mHLA}-\mathrm{DR} \mathrm{R}_{3}$ and $\triangle \mathrm{mHLA}-\mathrm{DR} \mathrm{R}_{7}$ were defined as the changes in $\mathrm{mHLA}-\mathrm{DR}$ value on day 3 and day 7 compared to that on day 0 . Data were compared between 28-day survivors and non-survivors. Receiver operating characteristic $(R O C)$ curves were plotted to measure the performance and discriminating threshold of $\triangle m H L A-D R_{3}, \triangle m H L A-D R_{7}, \triangle m H L A-D R_{7-3}, m H L A-D R_{0}, m H L A-D R_{3}$ and $\mathrm{mHLA}-\mathrm{DR} \mathrm{R}_{7}$ in predicting mortality of severe sepsis.

Results: ROC curve analysis showed that $\triangle \mathrm{mHLA}-D R_{3}$ and $\triangle \mathrm{mHLA}-\mathrm{DR} \mathrm{R}_{7}$ were reliable indicators of mortality in severe sepsis. A $\triangle \mathrm{mHLA}-\mathrm{DR} \mathrm{R}_{3}$ value of $4.8 \%$ allowed discrimination between survivors and non-survivors with a sensitivity of $89.0 \%$ and a specificity of $93.7 \%$; similarly, $\triangle \mathrm{mHLA}-\mathrm{DR} \mathrm{R}_{7}$ value of $9 \%$ allowed discrimination between survivors and non-survivors with a sensitivity of $85.7 \%$ and a specificity of $90.0 \%$. Patients with $\triangle \mathrm{mHLA}-\mathrm{DR} \mathrm{R}_{3} \leq 4.8 \%$ had higher mortality than those with $\triangle \mathrm{mHLA}-\mathrm{DR} \mathrm{R}_{3}>4.8 \%$ (71.4\% vs. 2.0\%, OR 125.00, 95\% Cl 13.93 to 1121.67); patients with $\triangle \mathrm{mHLA}-\mathrm{DR} \mathrm{R}_{7} \leq 9 \%$ had higher mortality than those with $\triangle \mathrm{mHLA}-\mathrm{DR} \mathrm{R}_{7}>9 \%$ (52.9\% vs. 2.0\%, OR 54.00, 95\% Cl 5.99 to 486.08). The mean change of mHLA-DR significantly increased in the survivor group with the passage of time; from day 0 to day 3 and day 7, changes were 6.45 and $16.90(P<0.05)$, respectively.
\end{abstract}

Conclusions: The change of $\mathrm{mHLA-DR}$ over time may be a reliable predictor for mortality in patients with severe sepsis.

\section{Introduction}

Severe sepsis is an important cause of admission to intensive care units (ICUs) throughout the world and is characterized by high mortality in adults [1-4]. But the pathogenesis of sepsis is still not clear. Previous reports suggest that the depression of the immune system may

\footnotetext{
* Correspondence: carlg@163.net

+ Contributed equally

'Department of Surgical Intensive Care Unit, The First Affiliated Hospital of

Sun Yat-sen University, 58 Zhongshan Er Road, Guangzhou 510080, PR China

Full list of author information is available at the end of the article
}

contribute to the severity of sepsis. Although the mechanistic and molecular bases for ICU-acquired immunosuppression are not exhaustively established, several features of the condition, including enhanced leukocyte apoptosis, lymphocyte anergy, and deactivated monocyte functions, have already been described [5-7]. mHLA-DR has been suggested to be a reliable marker for estimating immunosuppression. The level of mHLA-DR was significantly decreased during severe sepsis [8], although little is known about the underlying mechanism. The reduced expression

\section{Biomed Central}

(c) 2011 Wu et al.; licensee BioMed Central Ltd. This is an open access article distributed under the terms of the Creative Commons Attribution License (http://creativecommons.org/licenses/by/2.0), which permits unrestricted use, distribution, and reproduction in any medium, provided the original work is properly cited. 
was associated with high mortality and had a predictive value for the prognosis of patients with sepsis [9-12] or the risk of secondary infection [13-16]. However, the association between low mHLA-DR and mortality in severe sepsis has been challenged [17]. These differences in findings may be partially explained by the fact that immune function is dynamically changing during the clinical course of severe sepsis. In this study, we monitored the expression of mHLA-DR during 1 week to evaluate the predictive power of serial determinations of mHLA-DR as a marker of mortality in severe sepsis. Our hypothesis was that $\triangle$ mHLA-DR would be more accurate than mHLADR in predicting 28-day mortality in severe sepsis.

\section{Material and methods Patients}

This prospective single-center study was conducted in a surgical ICU (SICU) with 12 beds of a tertiary, teaching hospital between June 2008 and August 2010. A total of 107 patients were enrolled. Written informed consent was obtained from the patients or, for patients unable to provide consent, from their closest relatives. The study protocol was approved by the ethics committee of the hospital.

\section{Inclusion criteria}

Inclusion criteria were (a) age of between 18 and 85 years and (b) admission to the SICU with a diagnosis of severe sepsis according to criteria of the American College of Chest Physicians/Society of Critical Care Medicine [18]. Exclusion criteria were (a) being pregnant or lactating; (b) receiving immunosuppressive therapy such as cyclosporine, azothioprine, or cancer-related chemotherapy; (c) expected survival of fewer than 28 days because of incorrectable medical conditions, such as poorly controlled neoplasm or other end-stage disease; (d) history of bone marrow, lung, liver, kidney, pancreas, or small bowel transplantation; and (e) acute pancreatitis with no established source of infection.

\section{Study design}

Patients were screened on the admission day, and clinical and biological variables were collected. These included demographic characteristics (age and gender), microbiological findings (primary infection source and the identified microorganisms), and comorbidities (chronic obstructive pulmonary disease, chronic heart failure, malignant diseases, and diabetes). The primary outcome variable was mortality at day 28 (death or survival). The following clinical parameters were recorded: the initial severity as assessed by the Acute Physiology and Chronic Health Evaluation II (APACHE II) [19] and the Sequential Organ Failure Assessment (SOFA) (score range of 0 to 24) [20] on days 0 (on the day of ICU admission), 3, and 7. Blood samples for analysis of mHLA-DR were collected on days
0,3 , and 7 , respectively. $\triangle$ mHLA-DR ${ }_{3}$ and $\triangle$ mHLA-DR 7 were defined as the value change in mHLA-DR on days 3 and 7 compared with that on day 0 (mHLA-DR $)_{0}$ ), and $\triangle$ mHLA-DR ${ }_{7-3}$ was defined as the value change in mHLADR on day 7 compared with that on day 3. That is, $\triangle$ mHLA-DR $_{3}=$ mHLA-DR $3-$ mHLA-DR $; \Delta$ mHLA-DR $=$ mHLA-DR - mHLA-DR $;$ and $\Delta$ mHLA-DR ${ }_{7-3}=$ mHLA-DR - mHLA-DR 3 .

\section{mHLA-DR measurement by flow cytometry}

Expression of cell surface HLA-DR on monocytes was measured by flow cytometry (EPICSXL; Beckman Coulter, Inc., Fullerton, CA, USA). Staining and cell acquisition for flow cytometry were performed within 1 hour after blood sampling. Monoclonal antibodies were used as follows: CD14-PE $(20 \mu \mathrm{L}$, clone M5E2; BD Biosciences, San Jose, CA, USA) and HLA-DR-FITC ( $20 \mu \mathrm{L}$, clone G46-6; BD Biosciences) per $100 \mu \mathrm{L}$ of whole blood. Negative controls were mouse monoclonal antibodies IgG2a-PE (clone G155-178) and IgG2a-FITC (clone G155-178), which were isotype-matched in accordance with the recommendations of the manufacturer. Monocytes were characterized on the basis of their CD14 expression. At least 1,500 monocytes were analyzed from each sample. Results are expressed as percentages of HLA-DR-positive monocytes out of the total monocyte population.

\section{Statistical analysis}

Quantitative variables with normal distribution were expressed as mean \pm standard deviation, and quantitative variables with non-normal distribution were expressed as median and interquartile range (IQR). To estimate mean changes in mHLA-DR for the survivor and non-survivor groups, as well as corresponding between-group differences of changes in mHLA-DR, linear mixed models with random patient effects were employed. This analysis took into account the clustering of repeated measures in patients and the baseline (day 0) mHLA-DR and included all available cases. The survival estimate was based on the Kaplan-Meier method, and comparisons of survival distributions were based on the log-rank test. Receiver operating characteristic (ROC) curves were produced for $\triangle$ mHLA-DR $3, \Delta$ mHLA-DR,$\Delta$ mHLA-DR $7-3$, mHLA$\mathrm{DR}_{0}$, mHLA-DR 3 , and mHLA-DR 7 to determine the discriminating threshold of each parameter. The optimal cutoff points were determined by maximizing the sum of sensitivity and specificity. The areas under the ROC curves ( \pm standard error) were calculated for each parameter, and the comparison between AUCs was conducted on 'fully paired' case samples in accordance with the non-parametric approach reported by DeLong and colleagues [21]. The Pearson chi-square test or Fisher exact test, as appropriate, was used for categorical data. 
Univariate and multivariate logistic regressions were used to identify the variables associated with the risk of death assessed by odds ratios (ORs) and their 95\% confidence intervals (CIs). The variables with a $P$ value of not more than 0.10 in univariate analysis were entered in the multivariate adjusted model. The predictors included demographic and clinical parameters. Multivariate regression analysis was then performed for the independent variables by 'enter' method. Given that different $\triangle$ mHLADRs were not independent variables, they were entered into the model separately. A $P$ value of less than 0.05 was considered statistically significant. The analyses were performed with SPSS software (version 15.0; SPSS Inc., Chicago, IL, USA).

\section{Results}

\section{Baseline and clinical characteristics of the patients}

During the study period, 107 patients with severe sepsis were admitted to the SICU. Six patients did not meet the entry criterion regarding age limitation, 20 patients met at least one exclusion criterion, and 2 patients were lost to follow-up (Figure 1). Overall, 79 patients were enrolled in the study (63 males and 16 females). The ages ranged from 23 to 75 years (average age of $61 \pm$ 13.6 years), APACHE II score within 24 hours of SICU admission was a median of 19 (IQR 8), and the median SOFA score was 8 (IQR 5). The most common sites of infection were lungs and abdomen, accounting for 36 patients (45.6\%) and 42 patients (53.2\%), respectively. After admission, 7 patients died within 3 days and 13 other patients died within 7 days, leaving 79, 72, and 66

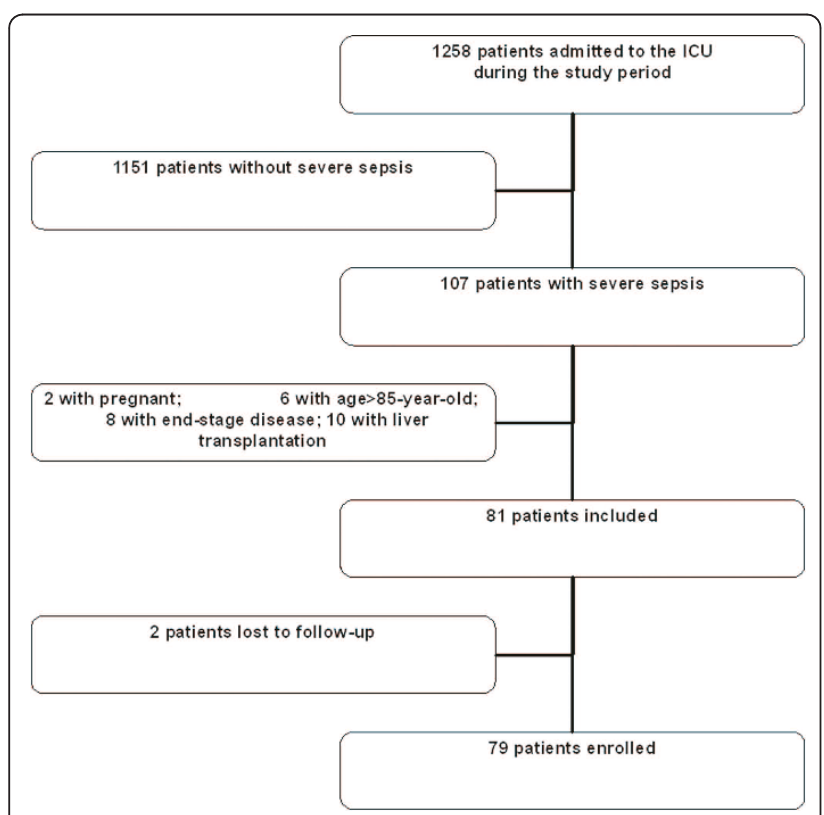

Figure 1 Trial profile. ICU, intensive care unit. surviving patients on days 0,3 , and 7 , respectively; overall 28-day mortality rate was $29.1 \%$ (23/79).

\section{Comparison of mHLA-DR expression changes between survivors and non-survivors}

mHLA-DR was significantly increased in the survivor group with the passage of time. The mean changes from day 0 to day 3 and day 7 were $6.45(P=0.002)$ and $16.90(P<0.001)$, respectively. It was $10.45(P<0.001)$ from day 3 to 7 . However, the non-survivor group presented no significant changes in mHLA-DR $(P>0.05)$. On day 3 , the difference of change from day 0 to 3 in mHLA-DR between survivors and non-survivors was 8.14 and was of borderline significance $(P=0.053)$. On day 7 , the corresponding differences were $12.35(P=$ $0.038)$ from day 0 to 7 and $4.21(P=0.423)$ from day 3 to 7 . These findings suggested that the survivors had a sustained increase in mHLA-DR over time and presented a significantly increasing tendency compared with the non-survivors (Table 1).

\section{Prognostic value of the changes of mHLA-DR expression}

The areas under the ROC curve (AUCs) of $\triangle$ mHLA-DR $_{3}$, $\triangle$ mHLA-DR ${ }_{7}$, and $\triangle$ mHLA-DR ${ }_{7-3}$ for 28-day mortality were $0.919(P<0.001), 0.938(P<0.001)$, and $0.729(P=$ 0.022 ), respectively; $\triangle$ mHLA-DR ${ }_{3}, \Delta$ mHLA-DR $_{7}$, and $\triangle$ mHLA-DR ${ }_{7-3}$ all had high specificity and sensitivity for prediction of mortality. The AUCs of mHLA-DR ${ }_{0}$, mHLA-DR ${ }_{3}$, and mHLA-DR 7 were $0.570(P>0.05), 0.629$ $(P>0.05)$, and $0.598(P>0.05)$, respectively. mHLA-DR ${ }_{0}$, mHLA-DR ${ }_{3}$, and mHLA-DR ${ }_{7}$ all had low specificity for prediction of mortality but had high sensitivity (Table 2 and Figures 2 and 3). The difference in AUC between $\triangle$ mHLA-DR $_{3}$ and mHLA-DR 3 is $0.289 \pm 0.077$ (95\% CI 0.139 to $0.439, P<0.001$ ), the difference in AUC between $\triangle$ mHLA-DR ${ }_{7}$ and mHLA-DR ${ }_{7}$ is $0.350 \pm 0.091(95 \%$ CI 0.172 to $0.528, P<0.001$ ), and the difference between $\triangle$ mHLA-DR $_{7-3}$ and mHLA-DR ${ }_{7}$ is $0.140 \pm 0.110(95 \% \mathrm{CI}$ -0.076 to $0.356, P=0.204)$.

There was a significant difference in mortality between patients with $\triangle$ mHLA-DR 3 of not more than $4.8 \%(15 / 21)$ and those with $\triangle$ mHLA-DR ${ }_{3}$ of greater than $4.8 \%(1 / 51)$ (71.4\% versus $2.0 \%$, OR $125.00,95 \%$ CI 13.93 to $1,121.67$ ). Similarly, patients with $\triangle$ mHLA-DR 7 of not more than $9 \%$ had a higher mortality rate (9/17) than those with $\triangle$ mHLA-DR $_{7}$ of greater than $9 \%(1 / 49)$ (52.9\% versus $2.0 \%$, OR $54.00,95 \%$ CI 5.99 to 486.08 ), and patients with $\triangle$ mHLA-DR ${ }_{7-3}$ of not more than $3.5 \%$ had a higher mortality rate $(8 / 28)$ than those with $\triangle$ mHLA-DR ${ }_{7-3}$ of greater than $3.5 \%(2 / 38)(28.6 \%$ versus $5.3 \%$, OR $7.20,95 \%$ CI 1.39 to 37.23$)$.

Gender and all $\triangle$ mHLA-DRs were entered in the multivariate regression analysis because their $P$ values were lower than 0.1 in univariate analysis. Given that different 
Table 1 Linear mixed model on the mean (95\% confidence interval) changes in mHLA-DR at every measure point in the survivor and non-survivor groups

\begin{tabular}{|c|c|c|c|c|c|c|c|c|c|}
\hline & \multicolumn{2}{|c|}{ Day 0} & \multicolumn{2}{|c|}{ Day 3} & \multicolumn{2}{|c|}{ Day 7} & \multirow[b]{2}{*}{$\Delta \mathrm{mHLA}-\mathrm{DR}_{3}$} & \multirow[b]{2}{*}{$\Delta \mathrm{mHLA}^{-\mathrm{DR}_{7}}$} & \multirow[b]{2}{*}{$\Delta \mathrm{mHLA}-\mathrm{DR}_{7-}$} \\
\hline & Number & mHLA-DR & Number & mHLA-DR & Number & mHLA-DR & & & \\
\hline Survivor & 56 & $\begin{array}{c}62.97 \\
(56.38 \sim 69.55)\end{array}$ & 56 & $\begin{array}{c}69.42 \\
(62.92 \sim 75.92)\end{array}$ & 56 & $\begin{array}{c}79.87 \\
(73.96 \sim 85.78)\end{array}$ & $\begin{array}{c}6.45^{\mathrm{a}} \\
(2.50 \sim 10.40)\end{array}$ & $\begin{array}{c}16.90^{\mathrm{b}} \\
(11.85 \sim 21.95)\end{array}$ & $\begin{array}{c}10.45^{c} \\
(6.19 \sim 14.71)\end{array}$ \\
\hline Non-survivor & 23 & $\begin{array}{c}56.77 \\
(46.49 \sim 67.05)\end{array}$ & 16 & $\begin{array}{c}55.08 \\
(44.26 \sim 65.90)\end{array}$ & 10 & $\begin{array}{c}61.32 \\
(49.79 \sim 72.84)\end{array}$ & $\begin{array}{c}-1.69^{d} \\
(-8.95 \sim 5.57)\end{array}$ & $\begin{array}{c}4.55^{\mathrm{e}} \\
(-5.96 \sim 15.06)\end{array}$ & $\begin{array}{c}6.24^{\dagger} \\
(-3.28 \sim 15.76)\end{array}$ \\
\hline $\begin{array}{l}\text { Between- } \\
\text { groups difference }\end{array}$ & & & & & & & $\begin{array}{c}8.14^{9} \\
(-0.12 \sim 16.40)\end{array}$ & $\begin{array}{c}12.35^{\mathrm{h}} \\
(0.70 \sim 24.00)\end{array}$ & $\begin{array}{c}4.21^{i} \\
(-6.21 \sim 14.64)\end{array}$ \\
\hline
\end{tabular}

$\triangle m H L A-D R_{3}$ and $\triangle m H L A-D R_{7}$ were defined as the value change in mHLA-DR on days 3 and 7 compared with that on day 0 , and $\triangle m H L A-D R_{7-3}$ was defined as the value change in $\mathrm{mHLA}-\mathrm{DR}$ on day 7 compared with that on day 3 . In the comparison of mean mHLA-DR differences in the same group between every measurement point, $P$ values were ${ }^{\mathrm{a}} 0.002,{ }^{\mathrm{b}}<0.001,{ }^{\mathrm{c}}<0.001,{ }^{\mathrm{d}} 0.644,{ }^{\mathrm{e}} 0.392$, and ${ }^{\mathrm{f}} 0.195$. In the comparison of $\Delta$ mHLA-DR differences between two groups within the same measurement point, $P$ values were ${ }^{9} 0.053,{ }^{h} 0.038$, and ${ }^{i} 0.423$. Cl, confidence interval; mHLA-DR, monocyte human leukocyte antigen-DR.

$\triangle$ mHLA-DRs were correlated with each other (a circumstance that would lead to collinearity if they were in the same model), different $\Delta$ mHLA-DRs were entered into the multivariate logistic regression model separately. After being adjusted for gender, the results indicated that $\triangle$ mHLA-DR $3(P<0.001), \Delta$ mHLA-DR $_{7}(P<$ $0.001)$, and $\triangle$ mHLA-DR ${ }_{7-3}(P=0.022)$ were associated with a higher mortality (Table 3 ).

\section{Comparison of 28-day mortality in patients grouped by mHLA-DR expression with $30 \%$ as a cutoff value} The 28-day mortality of patients with mHLA-DR expression of not more than $30 \%$ and those greater than $30 \%$ on days 0,3 , and 7 was compared, respectively. There was no significant difference in 28-day mortality between patients with mHLA-DR expression of not more than $30 \%$ and those greater than $30 \%$ on day 0 (50\% (5/10) versus $26.1 \%(18 / 69), P=0.120)$, day 3 (37.5\% (3/8) versus $20.3 \%(13 / 64), P=0.364)$, and day 7 (25.0\% (1/4) versus $14.5 \%(9 / 62), P=0.490)$, although non-survivors tended to exhibit lower mHLA-DR expression than survivors.

\section{Discussion}

Sepsis is one of the 10 leading causes of death in critically ill patients in the US. It is the third leading cause of death among patients in non-coronary ICUs [22,23]. Severe sepsis develops each year in more than 750,000 people, 215,000 of whom die of the disease [3], and is considered a disorder partly due to immunosuppression [5]. The diagnosis of immunosuppression depends on paraclinical parameters because of the absence of specific clinical symptoms. Among these, monocyte expression of human leukocyte antigen type DR (HLA-DR) has been shown to be useful for monitoring immunoparalysis and accepted as a reliable marker for evaluation of immune function $[8,15,17]$. Also, downregulation of mHLA-DR is generally accepted as a reliable marker for an immune dysfunction in patients with sepsis [24].

Volk and colleagues [12] were the first to describe immunoparalysis indicated by mHLA-DR expression in patients with sepsis. Abundant research has demonstrated the reduction of mHLA-DR expression in patients with sepsis $[8,9,13]$. A recent study indicated that patients in severe trauma present with a transient immunosuppression with decreased mHLA-DR expression. The lack of mHLA-DR recovery between days 3 and 4 and days 1 and 2 is associated with sepsis [25]. Furthermore, the prognostic value of low HLA-DR expression on monocytes has been elucidated, and the severity of the sepsis and mortality have been correlated with low HLA-DR expression $[8,10,15,26,27]$. In recent years, it has been shown that patients with sepsisinduced immunosuppression were at a higher risk to

Table 2 Predictive value for 28-day mortality of $\triangle$ mHLA-DR and mHLA-DR

\begin{tabular}{|c|c|c|c|c|c|c|c|c|c|}
\hline $\begin{array}{l}\text { Number of } \\
\text { patients }\end{array}$ & Variables & $\begin{array}{l}\text { Cutoff } \\
\text { value }\end{array}$ & Sensitivity & Specificity & $\begin{array}{c}\text { Positive } \\
\text { predictive value }\end{array}$ & $\begin{array}{c}\text { Negative } \\
\text { predictive value }\end{array}$ & AUC & $95 \% \mathrm{Cl}$ & $P$ value \\
\hline 72 & $\Delta \mathrm{mHLA}-\mathrm{DR}_{3}$ & $4.8 \%$ & $89.0 \%$ & $93.7 \%$ & $70.9 \%$ & $98.0 \%$ & $0.919 \pm 0.032$ & $0.83-0.97$ & $<0.001$ \\
\hline 66 & $\Delta \mathrm{mHLA}-\mathrm{DR}_{7}$ & $9.0 \%$ & $85.7 \%$ & $90.0 \%$ & $60.5 \%$ & $97.2 \%$ & $0.938 \pm 0.030$ & $0.851-0.982$ & $<0.001$ \\
\hline 66 & $\begin{array}{l}\Delta \mathrm{mHLA}- \\
\mathrm{DR}_{7-3}\end{array}$ & $3.5 \%$ & $66.1 \%$ & $80.0 \%$ & $37.1 \%$ & $92.9 \%$ & $0.729 \pm 0.079$ & $0.573-0.884$ & 0.022 \\
\hline 79 & $\mathrm{mHLA}-\mathrm{DR}_{0}$ & $35.0 \%$ & $89.1 \%$ & $43.5 \%$ & $62.1 \%$ & $79.3 \%$ & $0.570 \pm 0.070$ & $0.453-0.682$ & 0.319 \\
\hline 72 & $\mathrm{mHLA}-\mathrm{DR}_{3}$ & $39.5 \%$ & $91.1 \%$ & $37.5 \%$ & $54.6 \%$ & $83.6 \%$ & $0.629 \pm 0.075$ & $0.508-0.740$ & 0.116 \\
\hline 66 & $\mathrm{mHLA}^{-\mathrm{DR}_{7}}$ & $47.0 \%$ & $94.6 \%$ & $30.0 \%$ & $49.8 \%$ & $88.3 \%$ & $0.598 \pm 0.094$ & $0.460-0.708$ & 0.376 \\
\hline
\end{tabular}

$\triangle m H L A-D R_{3}$ and $\triangle m H L A-D R_{7}$ were defined as the value change in mHLA-DR on days 3 and 7 compared with that on day 0 , and $\triangle m H L A-D R_{7-3}$ was defined as the

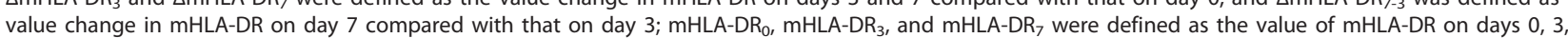
and 7. AUC, area under the curve; $\mathrm{Cl}$, confidence interval; mHLA-DR, monocyte human leukocyte antigen-DR. 

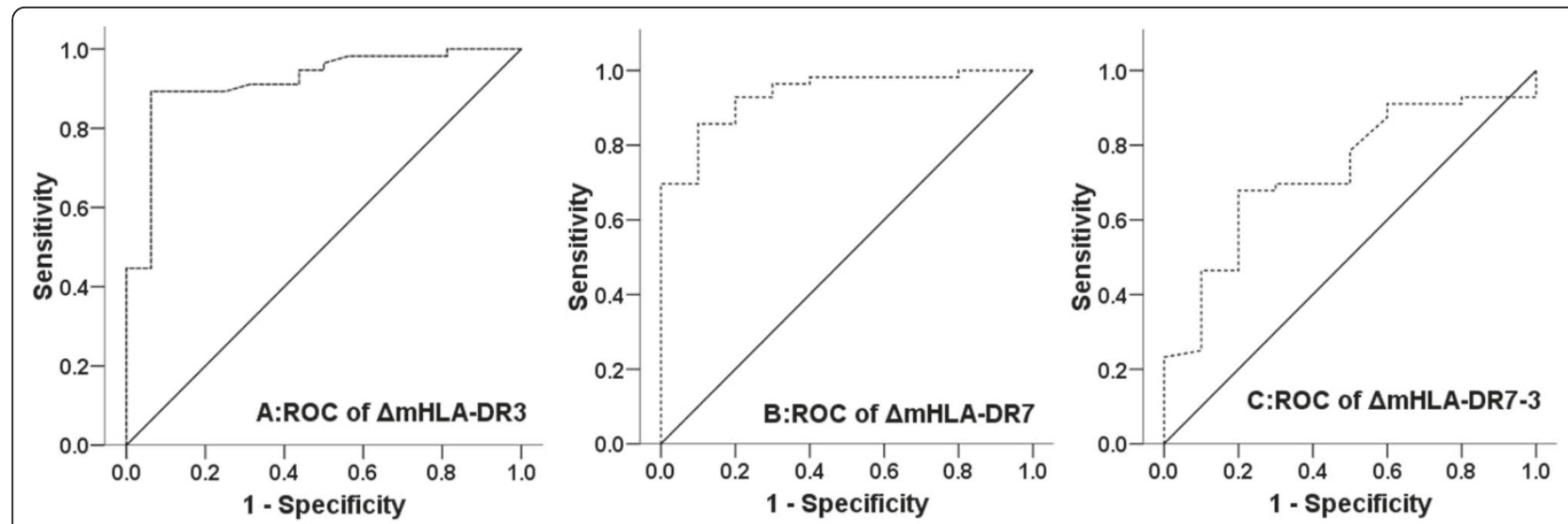

Figure 2 Receiver operating characteristic $(R O C)$ of $\triangle m$ mLA-DR,$\Delta m H L A-D R_{\mathbf{7}}$, and $\triangle$ mHLA-DR $_{\mathbf{7}-\mathbf{3}}$. ROC analysis depicted the discriminating value of (a) $\triangle m H L A-D R_{3}$, (b) $\triangle m H L A-D R_{7}$, and (c) $\triangle m H L A-D R_{7-3}$ for 28-day mortality in severe sepsis. $\triangle m H L A-D R_{3}$ and $\triangle m H L A-D R_{7}$ were defined as the value change in mHLA-DR on days 3 and 7 compared with that on day 0 , and $\triangle m H L A-D R_{7-3}$ was defined as the value change in mHLADR on day 7 compared with that on day 3. mHLA-DR, monocyte human leukocyte antigen-DR.

develop secondary infections [13]. In a prospective single-center observational trial, Landelle et al. found that persistently low mHLA-DR expression is independently associated with the development of nosocomial infections $[14,28]$. Monocyte HLA-DR expression has also been successfully applied to monitor immunomodulatory therapies, including medications such as granulocyte/macrophage colony-stimulating factor (GM-CSF) [29,30], filgrastim [31], thymosin alpha 1 [32], and interferon-gamma [33], as well as extracorporeal immune interventions such as immunoadsorption treatment and continuous hemodiafiltration [34,35].

In contrast, other research suggested that mHLA-DR was not a useful prognostic marker for outcome. A single-center study showed no significant difference in mortality between patients with low HLA-DR expression and those with normal HLA-DR expression [17]. That study indicated that mHLA-DR did not give satisfactory discriminatory power to assist in an outcome prediction. Another study reported that the low HLA-DR expression was not an independent outcome predictor, because the correlation between outcome and early HLA-DR expression disappeared after adjustment of the severity of illness by Sequential Organ Failure Assessment score or Simplified Acute Physiology Score II [13]. The contradictory results prompt clinicians to seek a more representative index for the connection between immune status and outcomes.

Although a number of recent studies have adopted static HLA-DR expression as a predictive marker, few of them have addressed the changes of HLA-DR expression during the disease progress. To address the fact

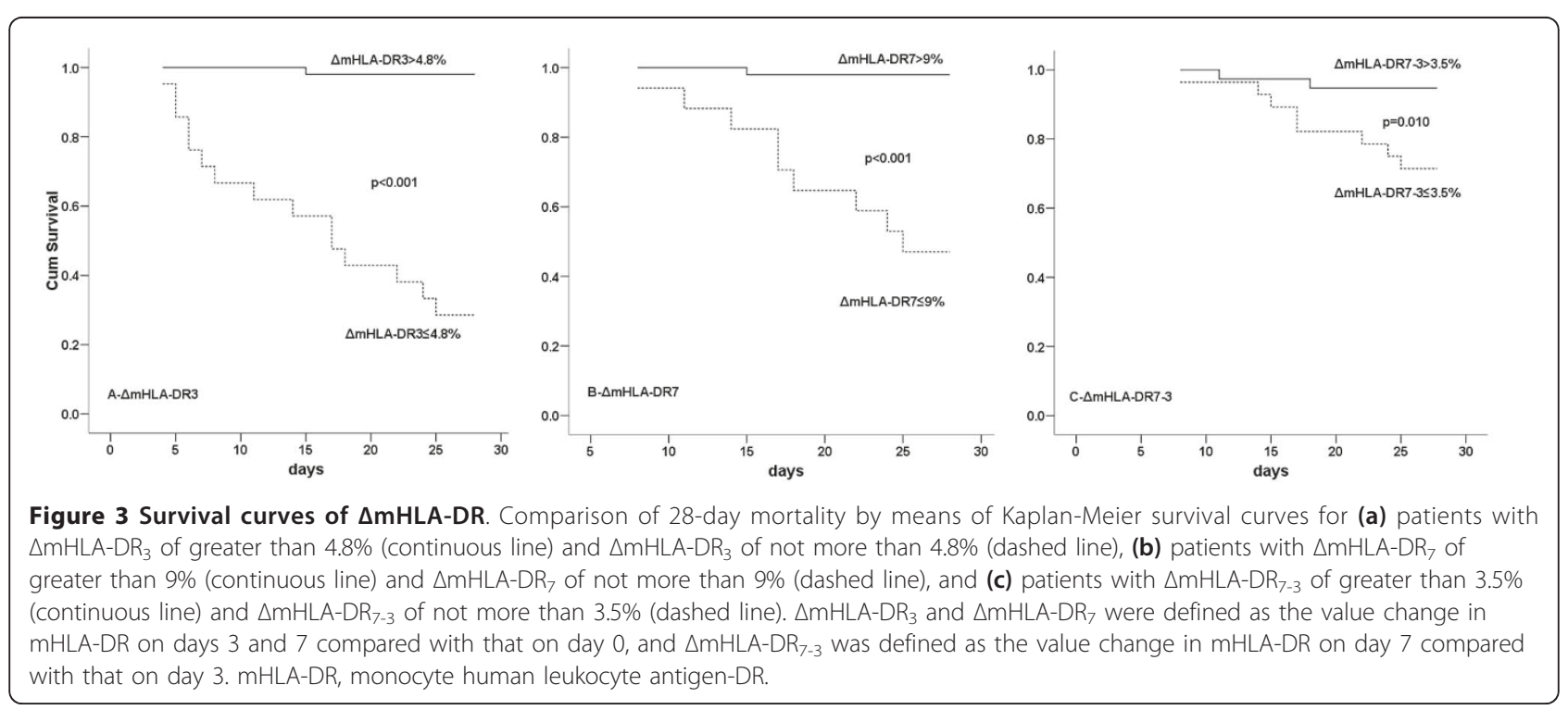


Table 3 Univariate and multivariate logistic regression analysis used to differentiate survivors and non-survivors

\begin{tabular}{|c|c|c|c|c|c|c|c|}
\hline & & \multicolumn{3}{|c|}{ Univariate $(n=66)$} & \multicolumn{3}{|c|}{ Multivariate $(n=66)$} \\
\hline & & OR & $95 \% \mathrm{Cl}$ & $P$ value & OR & $95 \% \mathrm{Cl}$ & $P$ value \\
\hline \multirow[t]{4}{*}{ Gender } & Male & 1.00 & - & - & & & \\
\hline & Female & 5.22 & $1.25-21.82$ & 0.023 & $8.19^{a}$ & $0.75-89.05$ & 0.084 \\
\hline & & & & & $4.56^{b}$ & $0.65-31.81$ & 0.126 \\
\hline & & & & & $5.47^{c}$ & $1.15-25.99$ & 0.033 \\
\hline \multirow[t]{2}{*}{ Age, years } & $\leq 62$ & 1.00 & - & - & & & \\
\hline & $>62$ & 2.51 & $0.59-10.69$ & 0.214 & & & \\
\hline \multirow{2}{*}{$\begin{array}{l}\text { APACHE ॥ } \\
\text { score }\end{array}$} & $\leq 18$ & 1.00 & - & - & & & \\
\hline & $>18$ & 2.53 & $0.63-9.89$ & 0.192 & & & \\
\hline \multirow[t]{2}{*}{ SOFA score } & $\leq 7$ & 1.00 & - & - & & & \\
\hline & $>7$ & 2.02 & $0.48-8.63$ & 0.341 & & & \\
\hline \multirow[t]{2}{*}{$\triangle \mathrm{mHLA}-\mathrm{DR}_{3}$} & $>4.8$ & 1.00 & - & - & & - & \\
\hline & $\leq 4.8$ & 75.00 & $8.04-699.44$ & $<0.001$ & $94.71^{a}$ & 7.64-1174.27 & $<0.001$ \\
\hline \multirow[t]{2}{*}{$\triangle \mathrm{mHLA}-\mathrm{DR}_{7}$} & $>9$ & 1.00 & - & - & & & \\
\hline & $\leq 9$ & 54.00 & $5.99-486.08$ & $<0.001$ & $51.04^{b}$ & $5.35-486.94$ & $<0.001$ \\
\hline \multirow[t]{2}{*}{$\triangle m H L A-D R_{7-3}$} & $>3.5$ & 1.00 & & & & & \\
\hline & $\leq 3.5$ & 7.20 & $1.39-37.23$ & 0.019 & $7.46^{c}$ & $1.34-41.39$ & 0.022 \\
\hline
\end{tabular}

${ }^{\mathrm{a}} \Delta \mathrm{mHLA}-\mathrm{DR}_{3},{ }^{\mathrm{b}} \Delta \mathrm{mHLA}-\mathrm{DR}_{7}$, and ${ }^{\mathrm{c}} \Delta \mathrm{mHLA}-\mathrm{DR}_{7-3}$ were entered into multivariate logistic regression model separately with adjustment of gender. $\triangle \mathrm{mHLA}-\mathrm{DR}{ }_{3}$ and $\triangle$ mHLA-DR $_{7}$ were defined as the value change in mHLA-DR on days 3 and 7 compared with that on day 0 , and $\triangle \mathrm{mHLA}^{-D R_{7-3}}$ was defined as the value change in mHLA-DR on day 7 compared with that on day 3. APACHE II, Acute Physiology and Chronic Health Evaluation II; Cl, confidence interval; OR, odds ratio; SOFA, Sequential Organ Failure Assessment.

that the immune function is dynamically influenced by severe sepsis and to compensate for the drawbacks of previous studies, we measured the expression of mHLADR consecutively to find out whether its change over time could predict mortality.

Our results indicated that mHLA-DR was significantly increased in the survivor group with the passage of time, but not in the non-survivor group. The findings were similar to those of Monneret and colleagues [27]. In a study in septic shock, they found that mHLA-DR expressions were not significantly different between survivors and non-survivors at days 1 and 2 . However, at days 3 and 4, the mHLA-DR expression had increased in survivors, but not in non-survivors.

We found that the AUCs of mHLA-DR 3 and mHLA$\mathrm{DR}_{7}$ for 28-day mortality in patients with severe sepsis were 0.629 and 0.598 , respectively, with low specificity despite the relatively high sensitivity. In contrast, $\triangle$ mHLADR was a good predictor for the outcome of severe sepsis. Among patients with severe sepsis, those with increased $\triangle$ mHLA-DR expression higher than threshold had markedly lower mortality. ROC curve analysis showed that $\triangle$ mHLA-DR ${ }_{3}$ and $\triangle$ mHLA-DR ${ }_{7}$ were reliable indicators of mortality in severe sepsis with high sensitivity and specificity. Multivariate logistic regression analysis showed that $\triangle$ mHLA-DR $3, \Delta$ mHLA-DR $_{7}$, and $\triangle$ mHLA-DR $_{7-3}$ were all associated with a higher mortality. However, the wide range of CIs implied poor precision because of the relatively small size of the cohort. It was also found that $\triangle$ mHLA-DR 7-3 $_{3}$ was not as good as $\triangle$ mHLA-DR $R_{7}$ and $\triangle$ mHLA-DR $R_{3}$ either in predicting mortality or in representing the difference in change of mHLA-DR between survivors and non-survivors. A possible explanation is that $\triangle$ mHLA-DR 3 and $\triangle$ mHLA-DR ${ }_{7}$ are calculated from a baseline on day 0 but that $\triangle$ mHLA-DR ${ }_{7-3}$ is calculated from a baseline on day 3 , meaning that $\triangle$ mHLA-DR 7-3 $_{3}$ is affected by more confounding factors such as the treatment in the ICU, the deterioration or improvement of disease, and other conditions that have impacts on immune status. Overall, our study suggests that elevated $\triangle$ mHLADR expression (especially $\triangle$ mHLA-DR ${ }_{3}$ and $\triangle$ mHLA$\mathrm{DR}_{7}$ ) may be seen as a marker for a gradually recovering immune function during the course of severe sepsis or a positive response to treatment and may indicate a better outcome.

A threshold of $30 \%$ is retained to predict mortality in published research that showed that non-survivors had an expression of mHLA-DR of lower than 30\% [9,36]. However, in this study, there was no significant difference in 28-day mortality between patients with mHLA-DR expression of not more than $30 \%$ and those greater than $30 \%$ on days 0,3 , and 7 , although non-survivors tended to exhibit lower mHLA-DR expression than survivors. At the same time, the present study found that mean expression of mHLA-DR in non-survivors was about $60 \%$. This finding may be explained by the following facts: (a) our study included only surgical patients, who may have a relatively minor degree of immunosuppression compared with other 
critical patients with severe underlying diseases, and (b) to ensure that severe sepsis was the most likely cause for patients' immunosuppression in our study, we had excluded severely immnunosuppressed patients caused by other factors, including post-transplantation status and immunosuppressive therapy. It is assumed that different patient populations and selection criteria as well as the heterogeneity of septic host [8] may contribute to the varied findings in different studies. Given the mixed results in different studies, a fixed static value of HLA-DR may not be appropriate to be applied in all patient populations and the dynamic change of HLA-DR seems more reasonable as an index for mortality.

The present study has certain limitations that need to be taken into account. This is a single-center study with a relatively small cohort, and the findings need to be confirmed by a multicenter study. In our study, mHLA-DR was expressed as percentages of HLA-DR-positive monocytes in the total monocyte population, and the measurement was reported to be reproducible with coefficients of variation from precision studies less than 5\% [37]. In spite of this, the value of delta HLA-DR should be separately interpreted because we cannot exclude the variability of measurements by flow cytometry. With the development of techniques, quantitative flow cytometry offers a better means of standardization within and between flow cytometers [38]. With this method, results become comparable among different laboratories.

Given these limitations, our objective is not to establish a golden standard about delta mHLA-DR for predicting severe sepsis mortality considering the small cohort and heterogeneity in severe sepsis but to remind clinicians that the dynamic change of mHLA-DR may be a better parameter than static values in judging prognosis and evaluating efficacy of immunomodulatory therapies. Actually, similar indices such as delta central venous pressure, delta stroke volume, and lactate clearance are being widely used in the ICU.

\section{Conclusions}

Our findings demonstrate that, in severe sepsis, monitoring the changes of mHLA-DR over time might be beneficial to predict mortality in comparison with a single measurement of mHLA-DR. This may help to identify patients at increased risk of death.

\section{Key messages}

- Whether mHLA-DR expression is a good predictor for mortality in patients with severe sepsis is controversial.

- In this prospective observational study, we monitored the changes of mHLA-DR expression in patients with severe sepsis.
- Our findings demonstrate that, in severe sepsis, monitoring the changes of mHLA-DR may be a better way to predict mortality than taking a single measurement of mHLA-DR.

\section{Abbreviations}

APACHE II: Acute Physiology and Chronic Health Evaluation II; AUC: area under curve; Cl: confidence interval; HLA-DR: human leukocyte antigen-DR; ICU: intensive care unit; IQR: interquartile range; mHLA-DR: monocyte human leukocyte antigen-DR; OR: odds ratio; ROC: receiver operating characteristic; SICU: surgical intensive care unit; SOFA: Sequential Organ Failure Assessment.

\section{Acknowledgements}

The authors gratefully thank Qing-Yu Kong (Department of Nephrology, Sun Yat-sen University) for excellent technical assistance. This work was supported by the Natural Science Foundation of Guangdong Province (8151008901000079 and 10451008901006286) and Sun Yat-sen University Clinical Research Program 5010 (2007015).

\section{Author details}

${ }^{1}$ Department of Surgical Intensive Care Unit, The First Affiliated Hospital of Sun Yat-sen University, 58 Zhongshan Er Road, Guangzhou 510080, PR China. ${ }^{2}$ Department of Medical Statistics and Epidemiology, School of Public Health, Sun Yat-sen University, 74 Zhongshan Er Road, Guangzhou 510080, PR China.

\section{Authors' contributions}

J-FW and JM designed and performed the research and wrote the manuscript. X-DG designed the research, provided the supportive work and supervision, and wrote the manuscript. JC, BO-Y, M-YC, L-FL, and Y-JL performed the research. A-HL analyzed the data. All authors read and approved the final manuscript.

\section{Competing interests}

The authors declare that they have no competing interests.

Received: 7 April 2011 Revised: 11 July 2011

Accepted: 20 September 2011 Published: 20 September 2011

\section{References}

1. Martin CM, Priestap F, Fisher H, Fowler RA, Heyland DK, Keenan SP, Longo CJ, Morrison T, Bentley D, Antman N: A prospective, observational registry of patients with severe sepsis: the Canadian Sepsis Treatment and Response Registry. Crit Care Med 2009, 37:81-88.

2. Heron M, Hoyert DL, Murphy SL, Xu J, Kochanek KD, Tejada-Vera B: Deaths: final data for 2006. Natl Vital Stat Rep 2009, 57:1-134.

3. Angus DC, Linde-Zwirble WT, Lidicker J, Clermont G, Carcillo J, Pinsky MR: Epidemiology of severe sepsis in the United States: analysis of incidence, outcome, and associated costs of care. Crit Care Med 2001, 29:1303-1310.

4. Cheng B, Xie G, Yao S, Wu X, Guo Q, Gu M, Fang Q, Xu Q, Wang D, Jin Y, Yuan S, Wang J, Du Z, Sun Y, Fang X: Epidemiology of severe sepsis in critically ill surgical patients in ten university hospitals in China. Crit Care Med 2007, 35:2538-2546.

5. Hotchkiss RS, Karl IE: The pathophysiology and treatment of sepsis. N Engl J Med 2003, 348:138-150.

6. Monneret G, Venet F, Pachot A, Lepape A: Monitoring immune dysfunctions in the septic patient: a new skin for the old ceremony. Mol Med 2008, 14:64-78.

7. Monneret G, Venet F, Kullberg BJ, Netea MG: ICU-acquired immunosuppression and the risk for secondary fungal infections. Med Mycol 2011, 49(Suppl 1):S17-23.

8. Gogos C, Kotsaki A, Pelekanou A, Giannikopoulos G, Vaki I, Maravitsa P, Adamis S, Alexiou Z, Andrianopoulos G, Antonopoulou A, Athanassia S, Baziaka F, Charalambous A, Christodoulou S, Dimopoulou I, Floros I, Giannitsioti E, Gkanas P, loakeimidou A, Kanellakopoulou K, Karabela N, Karagianni V, Katsarolis I, Kontopithari G, Kopterides P, Koutelidakis I, Koutoukas P, Kranidioti H, Lignos M, Louis K, et al: Early alterations of the 
innate and adaptive immune statuses in sepsis according to the type of underlying infection. Crit Care 2010, 14:R96.

9. Genel F, Atlihan F, Ozsu E, Ozbek E: Monocyte HLA-DR expression as predictor of poor outcome in neonates with late onset neonatal sepsis. $J$ Infect 2010, 60:224-228.

10. Ditschkowski M, Kreuzfelder E, Rebmann V, Ferencik S, Majetschak M, Schmid EN, Obertacke U, Hirche H, Schade UF, Grosse-Wilde H: HLA-DR expression and soluble HLA-DR levels in septic patients after trauma. Ann Surg 1999, 229:246-254.

11. Höflich C, Meisel C, Volk HD: The role and measurement of HLA-DR in septic patients. Adv Sepsis 2005, 4:134-139.

12. Volk HD, Thieme M, Heym S, Docke WD, Ruppe U, Tausch W, Manger D, Zuckermann S, Golosubow A, Nieter B: Alterations in function and phenotype of monocytes from patients with septic disease-predictive value and new therapeutic strategies. Behring Inst Mitt 1991, 208-215.

13. Lukaszewicz AC GM, Resche-Rigon M, Pirracchio R, Faivre V, Boval B, Payen D: Monocytic HLA-DR expression in intensive care patients: interest for prognosis and secondary infection prediction. Crit Care Med 2009, 37:2746-2752.

14. Landelle $\mathrm{C}$, Lepape A, Voirin $\mathrm{N}$, Tognet $\mathrm{E}$, Venet F, Bohe J, Vanhems $\mathrm{P}$, Monneret G: Low monocyte human leukocyte antigen-DR is independently associated with nosocomial infections after septic shock. Intensive Care Med 2010, 36:1859-1866.

15. Lekkou A, Karakantza M, Mouzaki A, Kalfarentzos F, Gogos CA: Cytokine production and monocyte HLA-DR expression as predictors of outcome for patients with community-acquired severe infections. Clin Diagn Lab Immunol 2004, 11:161-167.

16. Asadullah K, Woiciechowsky C, Docke WD, Egerer K, Kox WJ, Vogel S, Sterry W, Volk HD: Very low monocytic HLA-DR expression indicates high risk of infection-immunomonitoring for patients after neurosurgery and patients during high dose steroid therapy. Eur J Emerg Med 1995, 2:184-190.

17. Perry SE, Mostafa SM, Wenstone R, Shenkin A, McLaughlin PJ: Is low monocyte HLA-DR expression helpful to predict outcome in severe sepsis? Intensive Care Med 2003, 29:1245-1252.

18. Bone RC, Balk RA, Cerra FB, Dellinger RP, Fein AM, Knaus WA, Schein RM, Sibbald WJ: Definitions for sepsis and organ failure and guidelines for the use of innovative therapies in sepsis. The ACCP/SCCM Consensus Conference Committee. American College of Chest Physicians/Society of Critical Care Medicine. Chest 1992, 101:1644-1655.

19. Knaus WA, Draper EA, Wagner DP, Zimmerman JE: APACHE II: a severity of disease classification system. Crit Care Med 1985, 13:818-829.

20. Vincent JL, Moreno R, Takala J, Willatts S, De Mendonca A, Bruining $H$ Reinhart CK, Suter PM, Thijs LG: The SOFA (Sepsis-related Organ Failure Assessment) score to describe organ dysfunction/failure. On behalf of the Working Group on Sepsis-Related Problems of the European Society of Intensive Care Medicine. Intensive Care Med 1996, 22:707-710.

21. DeLong ER, DeLong DM, Clarke-Pearson DL: Comparing the areas under two or more correlated receiver operating characteristic curves: a nonparametric approach. Biometrics 1988, 44:837-845.

22. Martin GS, Mannino DM, Eaton S, Moss M: The epidemiology of sepsis in the United States from 1979 through 2000. N Engl J Med 2003, 348:1546-1554

23. Parrillo JE, Parker MM, Natanson C, Suffredini AF, Danner RL, Cunnion RE, Ognibene FP: Septic shock in humans. Advances in the understanding of pathogenesis, cardiovascular dysfunction, and therapy. Ann Intern Med 1990, 113:227-242.

24. Flohe S, Scholz M: HLA-DR monitoring in the intensive care unit-more than a tool for the scientist in the laboratory? Crit Care Med 2009, 37:2849-2850.

25. Cheron A, Floccard B, Allaouchiche B, Guignant C, Poitevin F, Malcus C, Crozon J, Faure A, Guillaume C, Marcotte G, Vulliez A, Monneuse O, Monneret G: Lack of recovery in monocyte human leukocyte antigen-DR expression is independently associated with the development of sepsis after major trauma. Crit Care 2010, 14:R208.

26. Frazier WJ, Hall MW: Immunoparalysis and adverse outcomes from critical illness. Pediatr Clin North Am 2008, 55:647-668.

27. Monneret G, Lepape A, Voirin N, Bohe J, Venet F, Debard AL, Thizy H, Bienvenu J, Gueyffier F, Vanhems P: Persisting low monocyte human leukocyte antigen-DR expression predicts mortality in septic shock. Intensive Care Med 2006, 32:1175-1183.
28. Schefold JC: Measurement of monocytic HLA-DR (mHLA-DR) expression in patients with severe sepsis and septic shock: assessment of immune organ failure. Intensive Care Med 2010, 36:1810-1812.

29. Meisel C, Schefold JC, Pschowski R, Baumann T, Hetzger K, Gregor J, WeberCarstens S, Hasper D, Keh D, Zuckermann H, Reinke P, Volk HD: Granulocyte-macrophage colony-stimulating factor to reverse sepsisassociated immunosuppression: a double-blind, randomized, placebocontrolled multicenter trial. Am J Respir Crit Care Med 2009, 180:640-648.

30. Nierhaus A, Montag B, Timmler N, Frings DP, Gutensohn K, Jung R, Schneider CG, Pothmann W, Brassel AK, Schulte Am Esch J: Reversal of immunoparalysis by recombinant human granulocyte-macrophage colony-stimulating factor in patients with severe sepsis. Intensive Care Med 2003, 29:646-651.

31. Schneider $C$, von Aulock $S$, Zedler $S$, Schinkel $C$, Hartung T, Faist E: Perioperative recombinant human granulocyte colony-stimulating factor (Filgrastim) treatment prevents immunoinflammatory dysfunction associated with major surgery. Ann Surg 2004, 239:75-81.

32. Lin HY: [Clinical trial with a new immunomodulatory strategy: treatment of severe sepsis with Ulinastatin and Maipuxin]. Zhonghua Yi Xue Za Zhi 2007, 87:451-457.

33. Docke WD, Randow F, Syrbe U, Krausch D, Asadullah K, Reinke P, Volk HD, Kox W: Monocyte deactivation in septic patients: restoration by IFNgamma treatment. Nat Med 1997, 3:678-681.

34. Schefold JC, von Haehling S, Corsepius M, Pohle C, Kruschke P, Zuckermann H, Volk HD, Reinke P: A novel selective extracorporeal intervention in sepsis: immunoadsorption of endotoxin, interleukin 6, and complement-activating product 5a. Shock 2007, 28:418-425.

35. Hirasawa H, Oda S, Matsuda K: Continuous hemodiafiltration with cytokine-adsorbing hemofilter in the treatment of severe sepsis and septic shock. Contrib Nephrol 2007, 156:365-370.

36. Volk HD, Reinke P, Krausch D, Zuckermann H, Asadullah K, Muller JM, Docke WD, Kox WJ: Monocyte deactivation-rationale for a new therapeutic strategy in sepsis. Intensive Care Med 1996, 22(Suppl 4): S474-481.

37. Monneret G, Elmenkouri N, Bohe J, Debard AL, Gutowski MC, Bienvenu J, Lepape A: Analytical requirements for measuring monocytic human lymphocyte antigen DR by flow cytometry: application to the monitoring of patients with septic shock. Clin Chem 2002, 48:1589-1592.

38. Docke WD, Hoflich C, Davis KA, Rottgers K, Meisel C, Kiefer P, Weber SU, Hedwig-Geissing M, Kreuzfelder E, Tschentscher P, Nebe T, Engel A, Monneret G, Spittler A, Schmolke K, Reinke P, Volk HD, Kunz D: Monitoring temporary immunodepression by flow cytometric measurement of monocytic HLA-DR expression: a multicenter standardized study. Clin Chem 2005, 51:2341-2347.

doi:10.1186/cc10457

Cite this article as: Wu et al:: Changes of monocyte human leukocyte antigen-DR expression as a reliable predictor of mortality in severe sepsis. Critical Care 2011 15:R220,

\section{Submit your next manuscript to BioMed Central and take full advantage of:}

- Convenient online submission

- Thorough peer review

- No space constraints or color figure charges

- Immediate publication on acceptance

- Inclusion in PubMed, CAS, Scopus and Google Scholar

- Research which is freely available for redistribution

Submit your manuscript at www.biomedcentral.com/submit
C Biomed Central 